See discussions, stats, and author profiles for this publication at: https://www.researchgate.net/publication/264237959

\title{
Erfassung von individualisiertem Unterricht in der Sekundarstufe I Eine Quantitative Überprüfung der Skala „Individualisierter Unterricht“ in zwei Schuluntersuchungen in der Steier...
}

Article in Zeitschrift für Bildungsforschung • November 2014

DOI: $10.1007 / 535834-014-0095-7$

\section{CITATIONS}

14

5 authors, including:

Markus Gebhardt

Universität Regensburg

129 PUBLICATIONS 981 CITATIONS

SEE PROFILE

Mathias Krammer

Pädagogische Hochschule Steiermark

20 PUBLICATIONS 203 CITATIONS

SEE PROFILE
(2) Susanne Schwab

University of Vienna

169 PUBLICATIONS 1,074 CITATIONS

SEE PROFILE

-7) Barbara Gasteiger-Klicpera

Karl-Franzens-Universität Graz

128 PUBLICATIONS 782 CITATIONS

SEE PROFILE

Some of the authors of this publication are also working on these related projects: 
Erfassung von individualisiertem Unterricht in der Sekundarstufe I Eine Quantitative Überprüfung der Skala "Individualisierter Unterricht" in zwei Schuluntersuchungen in der Steiermark Markus Gebhardt, Susanne Schwab, Mathias Krammer, Barbara GasteigerKlicpera \& Christine Sälzer

\section{Zeitschrift für Bildungsforschung}

ISSN 2190-6890

Z f Bildungsforsch

DOI 10.1007/s35834-014-0095-7

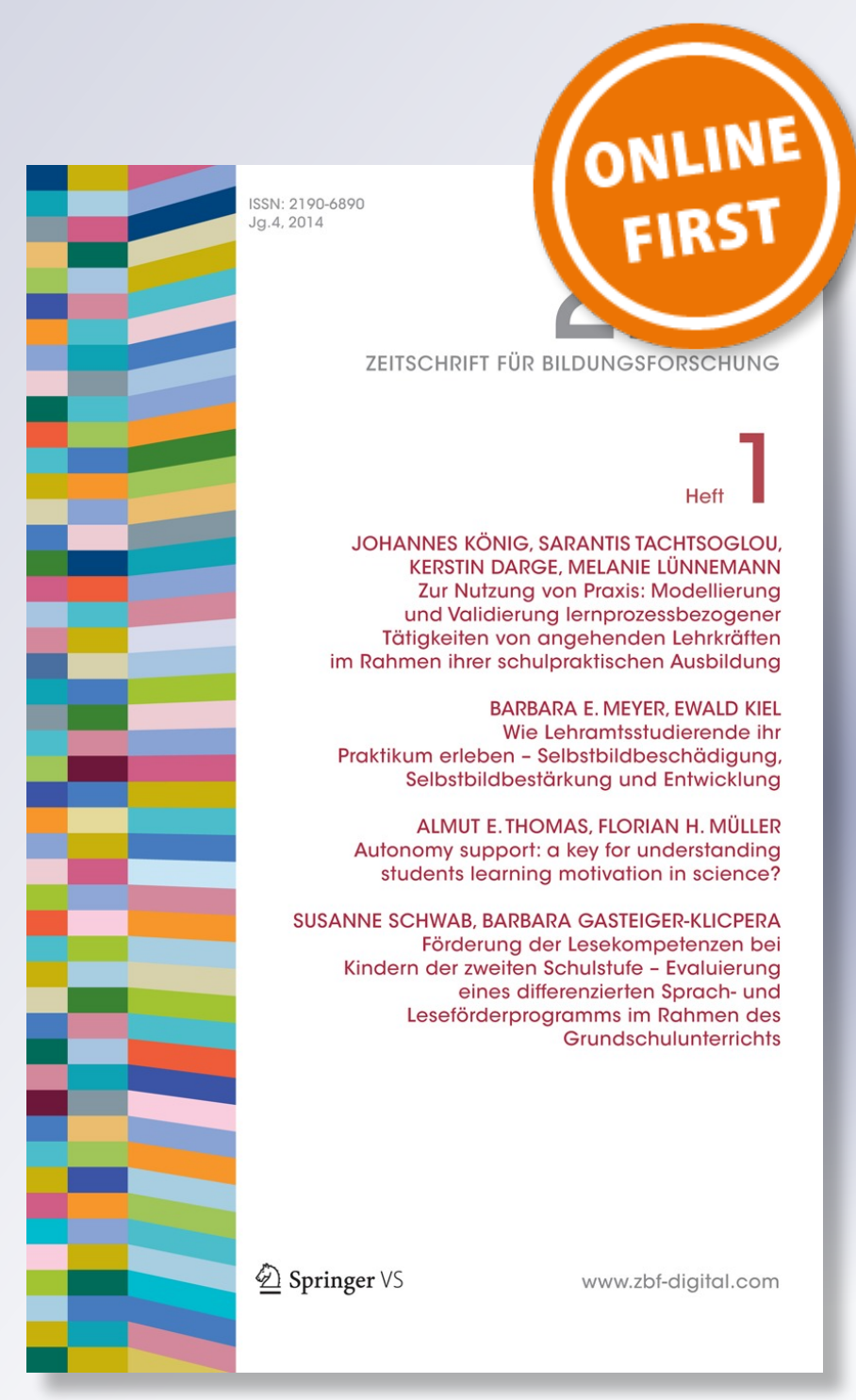

黛 Springer 
Your article is protected by copyright and all rights are held exclusively by Springer Fachmedien Wiesbaden. This e-offprint is for personal use only and shall not be selfarchived in electronic repositories. If you wish to self-archive your article, please use the accepted manuscript version for posting on your own website. You may further deposit the accepted manuscript version in any repository, provided it is only made publicly available 12 months after official publication or later and provided acknowledgement is given to the original source of publication and a link is inserted to the published article on Springer's website. The link must be accompanied by the following text: "The final publication is available at link.springer.com". 


\title{
Erfassung von individualisiertem Unterricht in der Sekundarstufe I Eine Quantitative Überprüfung der Skala „Individualisierter Unterricht" in zwei Schuluntersuchungen in der Steiermark
}

\author{
Markus Gebhardt · Susanne Schwab • Mathias Krammer • \\ Barbara Gasteiger-Klicpera $\cdot$ Christine Sälzer
}

\begin{abstract}
Zusammenfassung: Feyerer (1998) zeigte in den österreichischen Schulversuchen, dass integrative Schulkassen einen höheren Grad an individualisiertem Schulunterricht umsetzen als Klassen in der allgemeinen Schule ohne Schülerinnen und Schüler mit sonderpädagogischem Förderbedarf. Diese Aussage möchte die vorliegende Studie mehr als 10 Jahre später im integrativen Schulsystem der Steiermark mit der Skala zur Erfassung des individualisierten Unterrichts im integrativen Unterricht (Feyerer 1998) replizieren. In zwei Untersuchungen wurde die Skala erneut verwendet und empirisch überprüft. Dazu werden die Daten aus beiden Untersuchungen $(N=439$ Schülerinnen und Schüler der fünften und sechsten Schulstufe) analysiert. Die Ergebnisse einer Faktorenanalyse zeigten eine dreifaktorielle Faktorenlösung, welche den Ergebnissen von Feyerer (Behindern Behinderte? Integrativer Unterricht auf der Sekundarstufe I, 1998) nur in Teilen entspricht. Für eine neu konstruierte Kurzskala kann jedoch eine akzeptable interne Konsistenz (Cronbachs Alpha von 0,73) nachgewiesen werden. Ein Vergleich zwischen Integrations- und Regelschulklassen zeigte, dass Schülerinnen und Schüler aus Integrationsklassen den Unterricht
\end{abstract}

\footnotetext{
Angenommen: 14.04.2014

(C) Springer Fachmedien Wiesbaden 2014

Dr. M. Gebhardt $(\bowtie) \cdot$ Dr. C. Sälzer

TU München,

Arcisstraße 21,

80333 München, Deutschland

E-Mail: markus.gebhardt@tum.de

Dr. C. Sälzer

E-Mail: christine.saelzer@tum.de

Dr. S. Schwab · M. Krammer, M.A. · Univ.-Prof. Dr. B. Gasteiger-Klicpera

Universität Graz,

Merangasse 70 ,

8010 Graz, Österreich

E-Mail: susanne.schwab@uni-graz.at

M. Krammer, M.A.

E-Mail: mathias.krammer@uni-graz.at

Univ.-Prof. Dr. B. Gasteiger-Klicpera

E-Mail: barbara.gasteiger@uni-graz.at
} 
als integrativer wahrnahmen, als Schülerinnen und Schüler aus Regelschulklassen. Besonders die Antworten zur inneren Differenzierung wurden in Integrationsklassen häufiger positiv angegeben als in Regelschulklassen.

Schlüsselwörter: Individualisierter Unterricht · Integrationsklassen · Sonderpädagogische Förderbedarf

\section{Measuring teaching practices in inclusive settings in secondary classrooms a quantitative verification of the scale "individualized teaching" in two school surveys in Styria}

Summary: Feyerer (1998) points out that integrative classes show a higher level of individualized teaching than regular classes without students with Special Educational Needs in the Austrian School system. It was the main objective of this piece of research to validate this research finding from Feyerer. In order to do this, the scale "individualized teaching" from Feyerer was used in two studies with a total sample of 439 students. The results of a promax factor analysis show a three factor solution, a result which is not in accordance to the findings of Feyerer. However, the intern consistency of the new short scale was sufficient (Cronbach alpha: 0.73). Finally, a comparison between regular classes and integration classes shows that students from integrative classes perceive the teaching as more integrative than students from regular classes. In particular, questions regarding the internal differentiation were answered in a more positive way in integrative classes than in regular classes.

Keywords: Individualized teaching $\cdot$ Integration classes $\cdot$ Special educational needs

\section{Problemstellung}

Schulische Integration bezeichnet den gemeinsamen Unterricht von Schülerinnen und Schülern mit und ohne sonderpädagogischen Förderbedarf (SPF) in allgemeinbildenden Schulen. Im Schuljahr (2011/12) besuchten 52.23\% der österreichischen Schülerinnen und Schüler mit SPF integrativen Unterricht (Statistik Austria 2013). Das österreichische Bundesland Steiermark gilt als Vorreiterregion der Integration und beschult seit Jahren ca. 80\% der Kinder mit SPF in integrativen Klassen. Integration gilt hier folglich nicht mehr als Modellversuch oder Ausnahme, sondern als Regelfall. Spätestens seit der Umsetzung der UN-Konvention über die Rechte von Menschen mit Behinderungen (United Nations 2006) ist es das Ziel aller Bundesländer, Integration umzusetzen. Daher sollte schulische Integration nicht mehr nur in einzelnen Bundesländern wie der Steiermark die Norm darstellen.

\section{Integration und Inklusion}

Die beiden Begriffe Integration und Inklusion sind für den vorliegenden Artikel zentral. Hierbei geht der Gedanke der Inklusion weit über eine reine Platzierung des Kindes in einer Regelklasse hinaus. Denn in einem inklusiven Unterricht geht die Unterscheidung von Schülerinnen und Schülern durch einen SPF aufgrund der Beachtung der Bedürfnisse aller Schülerinnen und Schüler verloren (siehe auch „Zwei-Gruppen-Theorie“ von Hinz 
2002). Unter dem Begriff der Inklusion wird die Einbeziehung aller Schülerinnen und Schüler und deren Bedürfnisse ohne Unterschied im schulischen Leben verstanden (Bundschuh et al. 2002). Die Wahrnehmung der Lehrkräfte verschiebt sich somit von den beiden Gruppen hin zu den individuellen Bedürfnissen aller Schülerinnen und Schüler. Insofern ist Inklusion als eine optimierte Form der Integration zu verstehen, bei der alle Kinder mit ihren besonderen Bedürfnissen und Fähigkeiten eingeschlossen werden (Sander 2005). Ein Kernpunkt für die Umsetzung der UN-Konvention über die Rechte von Menschen mit Behinderungen (United Nations 2006) ist, dass der inklusive Unterricht als Regelform für die Beschulung aller Kinder angewendet wird (Gasteiger Klicpera und Wohlhart 2012). Infolge der Ratifizierung der UN-Konvention für Menschen mit Behinderung ist inklusiver Unterricht eine rechtliche Pflicht geworden und stellt nicht mehr ,nur“ eine Forderung von Elternverbänden und Betroffenen (Rutte und Schönwiese 2000) dar.

\section{Inklusiver Unterricht}

Inklusiver Unterricht ermöglicht allen Schülerinnen und Schülern ein erfolgreiches Lernen. Die Integrationsforschung wie auch die Inklusionsforschung beschäftigen sich schon seit vielen Jahren damit, was unter einem inklusiven Unterricht zu verstehen ist, wobei auf unterschiedliche Unterrichtskonzepte zurückgegriffen wurde (Seitz und Scheidt 2012). Jedoch wurde immer wieder klar, dass inklusiver Unterricht gleichsam individualisierter Unterricht sein muss (siehe dazu auch Mayr 2001). Effektive inklusive Beschulung, die alle Kinder in gleichem Ausmaß erreicht, ist eine große Herausforderung, welche nach Schulze (2013, S. 45) dem Prinzip der „Geeignetheit“ folgen muss. Inklusive Bildung muss die schulischen Bedingungen so adaptieren, dass diese den Bedürfnissen aller Schülerinnen und Schüler gerecht werden. Das Ziel ist nicht, dass Kinder mit SPF zur Schule passen, sondern die Schule sich an besondere Bedürfnisse anpassen können (Erten und Savage 2012). Dabei werden heterogenen Lernvoraussetzungen der Kinder als Bereicherung für das Unterrichtsgeschehen gesehen. Insofern erfordert inklusiver Unterricht einen hohen Anteil an offenen und kooperativen Lernformen, wie beispielsweise das Arbeiten mit einem Wochenplan, Stationslernen, Freiarbeit und Gesprächskreise (Heimlich 2007). Das vorrangige Ziel der Teilhabe aller Schülerinnen und Schüler am Unterricht wird durch die Individualisierung und innere Differenzierung des Unterrichts erreicht (Feyerer und Prammer 2003). Voraussetzung dafür ist, dass die Lehrerinnen und Lehrer den Kenntnis- und Wissensstand der einzelnen Schülerinnen und Schüler möglichst gut kennen und präsent haben. Als wichtigste Charakterisierung für einen inklusiven Unterricht wird die Überlegung und Denkweise der Lehrkraft gesehen (Dyson 2010). Diese Bedingung für einen inklusiven Unterricht ist sicherlich dem Zustand geschuldet, dass Inklusion situativ erfolgt und es eine Reihe an personenzentrierten, aber auch systemischen Einflussgrößen gibt, die je nach Einzelfall zu einer gelungenen Integration beitragen können oder nicht (Heimlich 2007). Welche Formen der Differenzierung die Lehrerinnen und Lehrer einsetzten, untersuchte Mayr (2001) in regulären Klassen der Sekundarstufe I. Hierbei gaben die Lehrpersonen an, einzelne einfache Differenzierungen häufig umzusetzen, während komplexere Formen eher selten angewendet wurden. Ebenso hing die Umsetzung der inneren Differenzierung 
von den personellen Ressourcen ab. So spielte es eine Rolle, ob die Lehrperson alleine für alle Schülerinnen und Schüler verantwortlich war oder ob im Team unterrichtet wurde.

\section{Erfassung von integrativen und inklusiven Unterrichtsmerkmalen}

Inklusive Schulen sind sehr verschieden. Dies zeigt sich beispielsweise an den letzten Schulpreisträgern des „Jakob Muth“-Preises für Inklusion. Dieser Preis wird seit dem Jahr 2009 jährlich an drei herausragende inklusive Schulen vergeben, welche bis auf den inklusiven Gedanken unterschiedliche Schulprofile und Ausgangslagen aufweisen. Diese Vielschichtigkeit von Inklusion ist für die schulische Praxis wunderbar, für die Operationalisierung in der Forschung jedoch nicht einfach zu lösen. Zwar kann die Erforschung der einzelnen inklusiven Schule sehr treffende Aussagen über die verschiedenen Aspekte der Inklusion hervorheben, allerdings bleibt die Aussagekraft beispielsweise über das Modell ,Fläming Schule‘ (Rosenberger 1998) oder das Modell ,Integrierte Gesamtschule Halle/Saale“ (Heimlich und Jacobs 2001) zu Gelingens- und Scheiternsfaktoren auf einer verallgemeinerbaren Ebene relativ gering. Daraus könnte man den Schluss ziehen, dass inklusive Schulen oder inklusiver Unterricht nicht empirisch mess- und vergleichbar sind. Dies wäre jedoch zu kurz gedacht, denn das Merkmal ,inklusiv` wurde bereits mehrfach untersucht. Am einfachsten geschieht dies durch eine kurzgefasste Definition über die Anwesenheit von Schülerinnen und Schülern mit SPF im gemeinsamen Unterricht (Dyson 2010). Hierbei fehlen Informationen über die Klasse und zusätzliche systemspezifische Ressourcen. Daher ist der zweite Weg Inklusion anhand der verschiedenen Beschulungsmodelle zu beobachten (Specht 1991). Aber auch dann kann man nicht Integration von Inklusion unterscheiden. Daher wird inklusiver Unterricht in der Forschung meist mit positiven Ausprägungen weiterer Konstrukte wie zum Beispiel Lehrerkooperation, Sozialklima der Klasse und der Anwendung von bestimmten Unterrichtsmethoden definiert. Ebenso wird Inklusion anhand der sozialen Partizipation von Schülerinnen und Schülern mit sonderpädagogischem Förderbedarf definiert (Dumke 1991; Preuss-Lausitz 1997; Klicpera und Gasteiger Klicpera 2003; Huber 2006; Huber und Wilbert 2012; Schwab et al. 2013). Zentrale Ergebnisse sind hier, dass die soziale Teilhabe aller Schülerinnen und Schüler gelingen kann (Dumke 1991; Preuss-Lausitz 1997; Feyerer 1998), jedoch in der Praxis Schülerinnen und Schüler mit SPF einem deutlichen Risiko der Ausgrenzung und Vereinsamung in integrativen Klassen ausgesetzt sein können (Klicpera und Gasteiger Klicpera 2003; Huber 2006; Huber und Wilbert 2012). Diese Studien definieren Inklusion somit nur indirekt über die soziale Teilhabe der Schülerinnen und Schüler mit SPF. Folglich können diese Untersuchungen zur Klärung der Wirkungsweise von inklusivem Unterricht nur eingeschränkt beitragen. Denn solange Inklusion nicht direkt und explizit definiert wird, können lediglich unspezifische Aussagen zum didaktischen Handeln gemacht werden. In diesem Beitrag wird der Begriff gemeinsamer Unterricht für die Anwesenheit von Schülerinnen und Schülern mit SPF im Unterricht verwendet, während der Begriff inklusiver Unterricht in Bezug auf eine gelungene Didaktik verstanden wird. 


\section{Erfassung der Individualisierung im Unterricht}

Feyerer (1998) wählte für die Evaluierung der österreichischen Schulversuche zur integrativen Beschulung in der Sekundarstufe einen Ansatz, bei dem die Didaktik im Mittelpunkt steht. In seiner Untersuchung bestimmten die Schülerinnen und Schüler den Inklusionsgrad des Unterrichts selbst. So beantworteten die Schülerinnen und Schüler neben Schulleistungstests auch Fragen zum Unterricht und zum sozialen Klima in ihrer Klasse. Um Inklusion zu messen, konstruierte Feyerer (1998) die Skala zur Erfassung der Individualisierung im Unterricht und verwendete zusätzlich Skalen zur Erfassung des Klassenklimas („Leistungsdruck“, „Schülerzentriertheit“, „Disziplin“, „Kohäsion“, „Sozialer Druck“ und „Vertrauen“) von Eder und Mayr (1994). Feyerer (1998) versteht unter Individualisierung eine Unterrichtsgestaltung, welche alle Schülerinnen und Schüler entsprechend ihrer individuellen Fähigkeiten fordert und fördert. Sofern Integrationsklassen die Prinzipien der inklusiven Pädagogik anwenden, sollte der Unterricht auch differenzierter und individualisierter gestaltet sein, welches in weiterer Folge zu einem positiveren Klassenklima führt. Für die Beurteilung der schulischen Umwelt stand die subjektive Wahrnehmung der Schülerinnen und Schüler im Vordergrund, welche den Unterricht und den Umgang mit ihren Lehrerinnen und Lehrern bewerten. Die Skala zur Erfassung des Individualisierungsgrades wurde theoretisch ausgehend von den Prinzipien der inklusiven Pädagogik sowie nach Helmkes (1988) Verständnis von „Adaptivität“ entwickelt. Aus zunächst 16 Items wurde mit 14 Items $(\alpha=0,73)$ die Skala „Individualisierter Unterricht“ gebildet, welche aus den Subskalen „Offene Lernformen“, „Unterschiedliche Aufgabenstellung“ und „Individuelles Eingehen“ besteht. Die Skala „Offene Lernformen“ $(\alpha=0,67)$ deckt mit vier Items Organisationsformen (Kleingruppenarbeit, Wochenplanarbeit, Freiarbeit und Projektunterricht) ab, welche überhaupt erst die Organisation von individualisiertem Unterricht ermöglichen. Die Skala „Unterschiedliche Aufgabenstellung “ $(\alpha=0,76)$ erfasst mit vier Items das Ausmaß des Eingehens der Lehrerinnen und Lehrer auf die individuellen Stärken und Schwächen der Schülerinnen und Schüler bei Aufgabenstellungen. Das „Individuelle Eingehen auf unterschiedliche Schülerinnen und Schüler" $(\alpha=0,75)$ bezieht sich mit sechs Items auf das Wahrnehmen und die Berücksichtigung der Heterogenität hinsichtlich der Leistungen, Interessen und Wünsche der Schülerinnen und Schüler. Dies beinhaltet das pädagogische Engagement einer persönlich-förderlichen Zuwendung sowie sorgendes und bemühtes Lehrerverhalten (Feyerer 1998). Feyerer (1998) verglich die schulische Umwelt von Integrationsklassen und Parallelklassen. Insgesamt konnte er dadurch zeigen, dass die Prinzipien einer inklusiven Pädagogik in Integrationsklassen in einem hohen Ausmaß gelebt werden. Hinsichtlich des Klassenklimas erlebten Schülerinnen und Schüler aus Integrationsklassen in allen Skalen ein positiveres Klima, wobei große Unterschiede in den Dimensionen „LehrerSchüler-Beziehungen“ und „Unterrichtsklima“ zu finden waren. Der größte Effekt bei den Schülerinnen und Schülern in den Integrationsklassen und den Regelschulklassen lag jedoch in dem Unterschied in der Skala „Individualisierter Unterricht“. 


\section{Fragestellung}

Im Vergleich zur Untersuchung von Feyerer im Jahr 1998 stellt Inklusion in der Steiermark inzwischen keinen Schulversuch sondern die Norm dar. So wird Integration in Österreich bundesweit meist in Integrationsklassen umgesetzt. Diese Klassen haben in den meisten Bundesländern bis zu 25 Kinder, in denen bis zu fünf Schülerinnen und Schüler mit SPF integriert werden. Anhand der Anzahl der Schülerinnen und Schüler mit SPF erfolgt eine Zuteilung der Lehrerstunden der Sonderpädagoginnen und Sonderpädagogen. Bei fünf Kindern mit SPF entspricht dies in der Steiermark 22 Lehrerstunden pro Woche (Gebhardt et al. 2012b).

Die vorliegende Studie untersucht die Bewertung des individualisierten Unterrichts von Schülerinnen und Schüler in integrativen Klassen und in Regelklassen. Hierbei kann es sein, dass die Parallelklassen ebenfalls hohe Werte in der Individualisierungsskala erzielen, da inklusive Prinzipien auch hier zum guten Unterricht gehören. Ebenso kann es aber auch sein, dass die inklusive Pädagogik in Integrationsklassen häufiger angewendet wird als in Parallelklassen. Insgesamt ist anzunehmen, dass im Vergleich zu den Schulversuchen und den Befunden von Feyerer (1998) sich der individualisierte Unterricht in den meisten Klassen durchgesetzt haben müsste, denn die Grundlagen des individualisierten Unterrichts werden seit Jahren in der Lehrerausbildung vermittelt und werden auch von der Schulbehörde gefordert (BMUKK 2008a; BMUKK 2008b; BMUKK 2009).

Es stellt sich daher die Frage, ob sich die Befunde von Feyerer (1998) in der Skala „Individualisierter Unterricht“ replizieren lassen. Dazu werden zunächst die Eigenschaften der Skala untersucht, bevor in einem zweiten Schritt auf inhaltliche Ergebnisse eingegangen wird.

\section{Methode}

In Rahmen zweier Studien (Schulische Integration im Längsschnitt - Kompetenz Entwicklung (SILKE), siehe dazu Gebhardt et al. 2012b; Studie zu Kooperativen Lernarrangements (KoopLEA), siehe dazu Gebhardt et al. 2012a) wurde die Skala „Individualisierter Unterricht" in der Sekundarstufe I in Graz eingesetzt. Beide Studien beschäftigen sich mit der schulischen Integration und verwenden ähnliche Längsschnittdesigns. Im Oktober und November 2011 wurden die teilnehmenden Klassen aus beiden Untersuchungen mittels Leistungstests und Fragebögen untersucht. Für die vorliegende Forschungsfrage war es insofern möglich, die Stichproben aus beiden Studien zusammenzuziehen, um eine größere Fallzahl zu erhalten. Zudem ermöglichte die Zusammenlegung der beiden Stichproben Schulen aus eher ländlichen Gegenden und Schulen aus städtischen Brennpunkten in die Untersuchung mit einzubeziehen.

\section{Stichprobe}

Insgesamt wurden 439 Schülerinnen und Schüler untersucht. Hiervon waren 194 (44.2\%) Mädchen und 245 (55.8\%) Jungen. 283 (64.5\%) kamen aus einer österreichischen Fami- 
lie und 154 (35.1\%) hatten einen Migrationshintergrund. In der 6. Schulstufe wurden im Rahmen der SILKE Studie 178 Schülerinnen und Schüler in 8 Integrationsklassen (Integrationsklassen 1-8) befragt und getestet. Hierbei hatten 35 Schülerinnen und Schüler einen sonderpädagogischen Förderbedarf. In der zweiten Studie (KoopLEA) wurden 260 Schülerinnen und Schüler aus 14 Klassen in der 5. Schulstufe untersucht. Hiervon waren 5 Klassen Integrationsklassen (Integrationsklassen 9-13) und 20 Schülerinnen und Schüler hatten einen SPF.

\section{Ergebnis zur Beschreibung des Instruments}

Für die Gesamtskala „Individualisierter Unterricht“ von Feyerer (1998) wurden alle 16 Items von Feyerer verwendet. Wegen der einfacheren Handhabbarkeit für die Schülerinnen und Schüler wurde die Skalierung an die Skalierungen der Fragebögen der SILKE und KoopLEA Studien angepasst (fünfstufig von 0 bis 4). Die Gesamtreliabilität der 16 Items erwies sich in dieser Befragung als wenig zufriedenstellend $(\alpha=0,61)$. Dies war auch nach der vorgeschlagenen Struktur von Feyerer (1998) mit 14 Items der Fall $(\alpha=0,62)$. Zudem sind die einzelnen Subskalen von Feyerer (1998) nicht reliabel (Offene Lernformen $(\alpha=0,27)$, Unterschiedliche Aufgabenstellung $(\alpha=0,49)$, Individuelles Eingehen auf die unterschiedliche Schülerinnen und Schüler $(\alpha=0,54))$.

Nachdem es sich bei der Skala um ein heterogenes Konstrukt handelt, das aus mehreren Subfaktoren bestehen kann, dessen Wirkweise in der Literatur unterschiedlich diskutiert wird (Dyson 2010; Heimlich 2007; Hinz 2002), wird in diesem Beitrag aus der bestehenden Skala „Individualisierung“ eine neue Kurzskala durch Itemreduzierung mittels Reliabilitätsanalyse gebildet. Die Durchführung erfolgte schrittweise. Dabei wurde in jedem Schritt das Item mit den schlechtesten psychometrischen Kennwerten aus der Skala entfernt.

Eine genaueren Analyse der Struktur der Skala konnte nicht durchgeführt werden, da eine Strukturanalyse mittels konfirmatorischer Faktorenanalyse aufgrund der Stichprobengröße nicht zulässig war.

Die mangelnde interne Konsistenz der einzelnen Skalen wurde insbesondere durch die negativ formulierten Items hervorgerufen. Vermutet werden kann, dass es insbesondere für Schülerinnen und Schüler mit SPF oder solche mit geringen Deutschkenntnissen schwierig war, die negativ gepolten Fragen zu verstehen. Nach der Itemreduzierung wurden folgende Items aus der Skala entfernt: „Unsere Lehrer verlangen sowohl von den guten als auch von den schlechten Schülern die gleichen Leistungen.“, „Meist tragen die Lehrer den Unterrichtsstoff von vorne vor.“ und „Unsere Lehrer wissen nicht, was uns Schüler interessiert“, sowie die Items „In unserer Klasse arbeiten wir oft in kleinen Gruppen“ und „Wir arbeiten oft mit einem Wochenplan“ und aus den folgenden Berechnungen ausgeschlossen.

Die neu konstruierte Kurzskala besteht aus drei Items der Subskala „Offene Lernformen“, drei Items der Subskala „Unterschiedliche Aufgabenstellung“ und fünf Items der Subskala „Individuelles Eingehen“. Die Reliabilität der Kurzskala $(\alpha=0,73)$ war ausreichend. Die Trennschärfen der Items $\left(r_{i t}=0,272-449\right.$; siehe Tab. 1) waren bei fast allen Items über dem Schwellenwert von 0,3 (Bühner 2011). Da aufgrund der veränderten 
Tab. 1: Faktorenstruktur und Trennschärfe der Skala „Individualisierter Unterricht“

\begin{tabular}{|c|c|c|c|c|}
\hline & $\begin{array}{l}\text { Fakor- } \\
\text { ladung I }\end{array}$ & $\begin{array}{l}\text { Fakor- } \\
\text { ladung II }\end{array}$ & $\begin{array}{l}\text { Fakor- } \\
\text { ladung III }\end{array}$ & $r_{i t}$ \\
\hline \multicolumn{5}{|l|}{$N$} \\
\hline \multicolumn{5}{|l|}{ Unterschiedliche Aufgabenstellung } \\
\hline $\begin{array}{l}\text { Schwächere Schüler bekommen leichtere } \\
\text { Aufgaben }\end{array}$ & 0,836 & 0,054 & 0,179 & 0,395 \\
\hline $\begin{array}{l}\text { Guten Schüler werden schwierigere Auf- } \\
\text { gaben gestellt }\end{array}$ & 0,849 & 0,144 & 0,137 & 0,449 \\
\hline $\begin{array}{l}\text { Unsere Lehrer wenden für die schwächeren } \\
\text { Schüler mehr Zeit auf }\end{array}$ & 0,550 & 0,381 & 0,151 & 0,411 \\
\hline $\begin{array}{l}\text { Projektunterricht findet in unserer Klasse } \\
\text { oft statt }\end{array}$ & 0,447 & 0,448 & $-0,102$ & 0,330 \\
\hline $\begin{array}{l}\text { Unsere Lehrer bieten den guten Schülern } \\
\text { zusätzliche Beispiele an }\end{array}$ & 0,418 & 0,412 & 0,211 & 0,379 \\
\hline \multicolumn{5}{|c|}{ Individuelles Eingehen auf unterschiedliche Schüler } \\
\hline $\begin{array}{l}\text { Wenn sich jemand nicht auskennt, findet er } \\
\text { einen Mitschüler der Ihm hilft }\end{array}$ & 0,039 & 0,621 & 0,394 & 0,340 \\
\hline $\begin{array}{l}\text { Unsere Lehrer wissen genau über die } \\
\text { Stärken und Schwächen jedes einzelnen } \\
\text { Schülers Bescheid }\end{array}$ & 0,214 & 0,563 & 0,343 & 0,383 \\
\hline $\begin{array}{l}\text { Auch wenn jemand die Antwort nicht gleich } \\
\text { weiß, lassen ihm die Lehrer genügend Zeit } \\
\text { zum Antworten }\end{array}$ & 0,172 & 0,685 & 0,124 & 0,366 \\
\hline $\begin{array}{l}\text { Unsere Lehrer bemühen sich sehr um jeden } \\
\text { einzelnen Schüler }\end{array}$ & 0,137 & 0,765 & 0,136 & 0,393 \\
\hline \multicolumn{5}{|l|}{ Schülerorientierung } \\
\hline $\begin{array}{l}\text { Oft dürfen wir Schüler selbst bestimmen, } \\
\text { was und wie wir lernen }\end{array}$ & 0,084 & 0,199 & 0,869 & 0,272 \\
\hline $\begin{array}{l}\text { Im Unterricht wird auf die Wünsche der } \\
\text { Schüler eingegangen }\end{array}$ & 0,361 & 0,329 & 0,666 & 0,432 \\
\hline \multicolumn{4}{|c|}{ Die Skalierung der Items war fünfstufig von 0 bis 4} & $\alpha=0,730$ \\
\hline
\end{tabular}

Zusammensetzung der Skala die ursprüngliche Struktur nicht mehr angenommen werden kann, wurde eine explorative Faktorenanalyse berechnet. Da hinter der Skala ein starker Generalfaktor vermutet wurde, wurde die Hauptkomponentenanalyse promax-rotiert (siehe dazu Bühner 2011). Die Heterogenität der Kurzskala zeigte sich auch in der Faktorenstruktur. Die promax-rotierte Hauptkomponentenanalyse $(K M O=0,742$; Bartletts Test $p<0,001)$ ergab drei Faktoren mit einem Eigenwert $>1$. Während der erste Faktor $27.45 \%$ der Varianz aufklärt, klärt Faktor II 14.14\% Varianz auf und die Varianzaufklärung des Faktors III beträgt nur mehr 9.85\%. Die Faktorenladungen der einzelnen Items sind in Tab. 1 angeführt.

Die Faktorenanalyse ergab ähnlich wie bei Feyerer (1998) eine dreifaktorielle Lösung als die am besten passende Faktorenstruktur. Nachdem die Faktoren nicht den Subskalen nach Feyerer (1998) entsprechen, werden die neuen Faktoren nachfolgend beschrieben. 
Faktor I erhielt die Bezeichnung „Unterschiedliche Aufgabenstellung“, da er neben den von Feyerer gebildeten drei Items zusätzlich die Aussage „Unsere Lehrer wenden für die schwächeren Schüler mehr Zeit auf“ und „Projektunterricht findet in unserer Klasse oft statt“ enthielt. Faktor II beinhaltet von der ursprünglichen Skala „Individuelles Eingehen“ drei Items und das zusätzliche Item „Wenn sich jemand nicht auskennt, findet er einen Mitschüler der ihm hilft." Faktor III entspricht nicht mehr dem von Feyerer (1998) gefundenen Faktor „offene Lernformen“, da deren weitere Items hier dem Faktor I zugeordnet wurden oder in der Itemreduzierung gelöscht wurden. Der hier gefundene Faktor III besteht aus zwei Items, welche bei Feyerer (1998) aus den Faktoren „Offene Lernformen“ und „Individuelles Eingehen“ stammen. Der hier gefundene Faktor III wird mit dem Begriff „Schülerorientierung“ beschrieben. Die nachfolgenden Ergebnisse der Items wurden nach dieser hier vorgefundenen Faktorenstruktur geordnet. Nachdem diese Faktoren nur explorativ in der vorliegenden Studie gebildet wurden, werden die Faktoren nur als Übersicht benutzt und nicht einzeln in die weiteren Berechnungen miteinbezogen.

\section{Ergebnisse auf Ebene der Schulklassen}

Tabelle 2 sind die Mittelwerte und Standardabweichungen der einzelnen Items für die vorliegenden Stichproben zu entnehmen.

Die deskriptive Auswertung der einzelnen Items zeigt, dass der empirische Mittelwert aller Items nahe am theoretischen Mittelwert der Skala $(M=2$ bei einer fünfstufigen Skala von 0 bis 4) lag. Eher zugestimmt haben die Schülerinnen und Schüler den Fragen bezogen auf die Aufgabenstellung und dem Miteinander in der Klasse, während die Fragen zur Schülerorientierung eher negativ beantwortet wurden. Zwischen den Schülerinnen und Schülern in Integrations- und Parallelklassen wurden t-Tests berechnet, um signifikante Gruppenunterschiede zu identifizieren (siehe Tab. 2). Die Schülerinnen und Schüler der Integrationsklassen gaben in den Items zur „Differenzierung der Aufgaben“, „,der Verwendung von Projektunterricht“, „,der Zusatz-Zeit für schwächere Schülerinnen und Schüler“ sowie bei dem Item zum „Schülerwunsch im Unterricht“ signifikant höhere Werte an. Die Schülerinnen und Schüler in den Parallelklassen zeigten hingegen bei dem Item „Hilfe von Mitschülern“ eine höhere Zustimmung.

Für die Skalenbildung der Skala „Individualisierter Unterricht“ wurden die Werte der einzelnen Items summiert. Das theoretische Skalenminimum liegt somit bei 0, das Maximum der Skala (11 Items) bei einem Wert von 44. Mit Hilfe einer einfaktoriellen univariaten Varianzanalyse wurde der Unterschied zwischen Schülerinnen und Schülern aus den Integrationsklassen und den Regelklassen überprüft. Die unabhängige Variable stellte die Klasse dar (Integrationsklasse 5. Schulstufe, Integrationsklasse 6. Schulstufe sowie die entsprechenden Regelschulklassen), die abhängige Variable war der Skalensummenwert. Das Ergebnis zeigten einen signifikanten Haupteffekt $(F(2,438)=5,35 ; p<0,01)$. Wie anhand des Post-hoc-Tests nach Bonferroni zu sehen war, unterschieden sich die Mittelwerte der Schülerinnen und Schüler der Integrationsklassen beider Schulstufen von jenen der Schülerinnen und Schüler in Parallelklassen gering signifikant $(S E=0,78$, $p=0,03 ; S E=0,96, p=0,01)$. 
Tab. 2: Mittelwerte und Standardabweichungen der einzelnen Items

\begin{tabular}{|c|c|c|c|c|}
\hline & $\begin{array}{l}\text { Integrations- } \\
\text { klassen } 5 . \\
\text { Schulstufe } \\
\end{array}$ & $\begin{array}{l}\text { Integrations- } \\
\text { klassen } 6 . \\
\text { Schulstufe }\end{array}$ & $\begin{array}{l}\text { Regelklassen } 6 . \\
\text { Schulstufe }\end{array}$ & $t$ \\
\hline$N$ & 178 & 88 & 173 & \\
\hline $\begin{array}{l}\text { Schwächere Schüler bekommen } \\
\text { leichtere Aufgaben }\end{array}$ & $\begin{array}{c}2,52 \\
(1,53)\end{array}$ & $\begin{array}{c}2,60 \\
(1,46)\end{array}$ & $\begin{array}{c}1,36 \\
(1,51)\end{array}$ & $-8,03 * *$ \\
\hline $\begin{array}{l}\text { Guten Schüler werden schwieri- } \\
\text { gere Aufgaben gestellt }\end{array}$ & $\begin{array}{c}2,60 \\
(1,41)\end{array}$ & $\begin{array}{c}2,61 \\
(1,34)\end{array}$ & $\begin{array}{c}1,77 \\
(1,60)\end{array}$ & $-5,58 * *$ \\
\hline $\begin{array}{l}\text { Unsere Lehrer wenden für die } \\
\text { schwächeren Schüler mehr Zeit } \\
\text { auf }\end{array}$ & $\begin{array}{c}2,55 \\
(1,28)\end{array}$ & $\begin{array}{c}2,59 \\
(1,12)\end{array}$ & $\begin{array}{l}2,27 \\
(1,39)\end{array}$ & $-2,24 *$ \\
\hline $\begin{array}{l}\text { Projektunterricht findet in unse- } \\
\text { rer Klasse oft statt }\end{array}$ & $\begin{array}{c}2,13 \\
(1,20)\end{array}$ & $\begin{array}{c}2,31 \\
(1,22)\end{array}$ & $\begin{array}{c}1,91 \\
(1,22)\end{array}$ & $-2,34 * *$ \\
\hline $\begin{array}{l}\text { Unsere Lehrer bieten den guten } \\
\text { Schülern zusätzliche Beispiele an }\end{array}$ & $\begin{array}{c}2,84 \\
(1,10)\end{array}$ & $\begin{array}{c}2,72 \\
(1,28)\end{array}$ & $\begin{array}{c}2,96 \\
(1,28)\end{array}$ & 1,38 \\
\hline $\begin{array}{l}\text { Wenn sich jemand nicht aus- } \\
\text { kennt, findet er einen Mitschü- } \\
\text { ler der Ihm hilft }\end{array}$ & $\begin{array}{c}2,71 \\
(1,24)\end{array}$ & $\begin{array}{c}2,57 \\
(1,35)\end{array}$ & $\begin{array}{c}3,05 \\
(1,04)\end{array}$ & $3,42 * *$ \\
\hline $\begin{array}{l}\text { Unsere Lehrer wissen genau } \\
\text { über die Stärken und Schwä- } \\
\text { chen jeden einzelnen Schüler } \\
\text { Bescheid }\end{array}$ & $\begin{array}{c}2,85 \\
(1,17)\end{array}$ & $\begin{array}{c}2,82 \\
(1,26)\end{array}$ & $\begin{array}{c}2,76 \\
(1,20)\end{array}$ & $-0,68$ \\
\hline $\begin{array}{l}\text { Auch wenn jemand die Antwort } \\
\text { nicht gleich weiß, lassen ihm } \\
\text { die Lehrer genügend Zeit zum } \\
\text { Antworten }\end{array}$ & $\begin{array}{c}2,83 \\
(1,22)\end{array}$ & $\begin{array}{c}2,85 \\
(1,20)\end{array}$ & $\begin{array}{l}2,75 \\
(1,15)\end{array}$ & $-0,72$ \\
\hline $\begin{array}{l}\text { Unsere Lehrer bemühen sich } \\
\text { sehr um jeden einzelnen } \\
\text { Schüler }\end{array}$ & $\begin{array}{c}3,07 \\
(1,11)\end{array}$ & $\begin{array}{c}3,41 \\
(1,07)\end{array}$ & $\begin{array}{l}3,31 \\
(0,99)\end{array}$ & 1,18 \\
\hline $\begin{array}{l}\text { Im Unterricht wird auf } \\
\text { die Wünsche der Schüler } \\
\text { eingegangen }\end{array}$ & $\begin{array}{c}1,53 \\
(1,22)\end{array}$ & $\begin{array}{c}1,57 \\
(1,23)\end{array}$ & $\begin{array}{c}1,24 \\
(1,20)\end{array}$ & $-2,61 * *$ \\
\hline $\begin{array}{l}\text { Oft dürfen wir Schüler selbst } \\
\text { bestimmen, was und wie wir } \\
\text { lernen }\end{array}$ & $\begin{array}{c}1,12 \\
(1,34)\end{array}$ & $\begin{array}{c}1,40 \\
(1,39)\end{array}$ & $\begin{array}{c}1,32 \\
(1,33)\end{array}$ & 0,816 \\
\hline Gesamt & $\begin{array}{l}26,75 \\
(7,25)\end{array}$ & $\begin{array}{l}27,44 \\
(7,63)\end{array}$ & $\begin{array}{l}24,70 \\
(7,17)\end{array}$ & \\
\hline
\end{tabular}

Skalierung von $0=$ "stimmt nie" bis $4=$,stimmt immer"

${ }^{*} p=0,05 ;{ }^{* *} p=0,01$

Um eine aussagekräftige Statistik für den Einfluss auf Ebene der Klassen zu erhalten, wurde für die Bewertung des individualisierten Unterrichts eine Mehrebenenanalyse „Random Effects Model“" (Snijders und Bosker 2011) berechnet. Dies bedeutet, dass die erhobenen Bewertungen der Schülerinnen und Schüler bezogen auf die Zugehörigkeit zu einer Klasse analysiert und verglichen wurden. Der Intraclass-Correlation Coefficient (ICC) zeigte, dass $15.89 \%$ (Wald $Z=2,55, p=0,01$ ) der Varianz zwischen den Klassen besteht, 


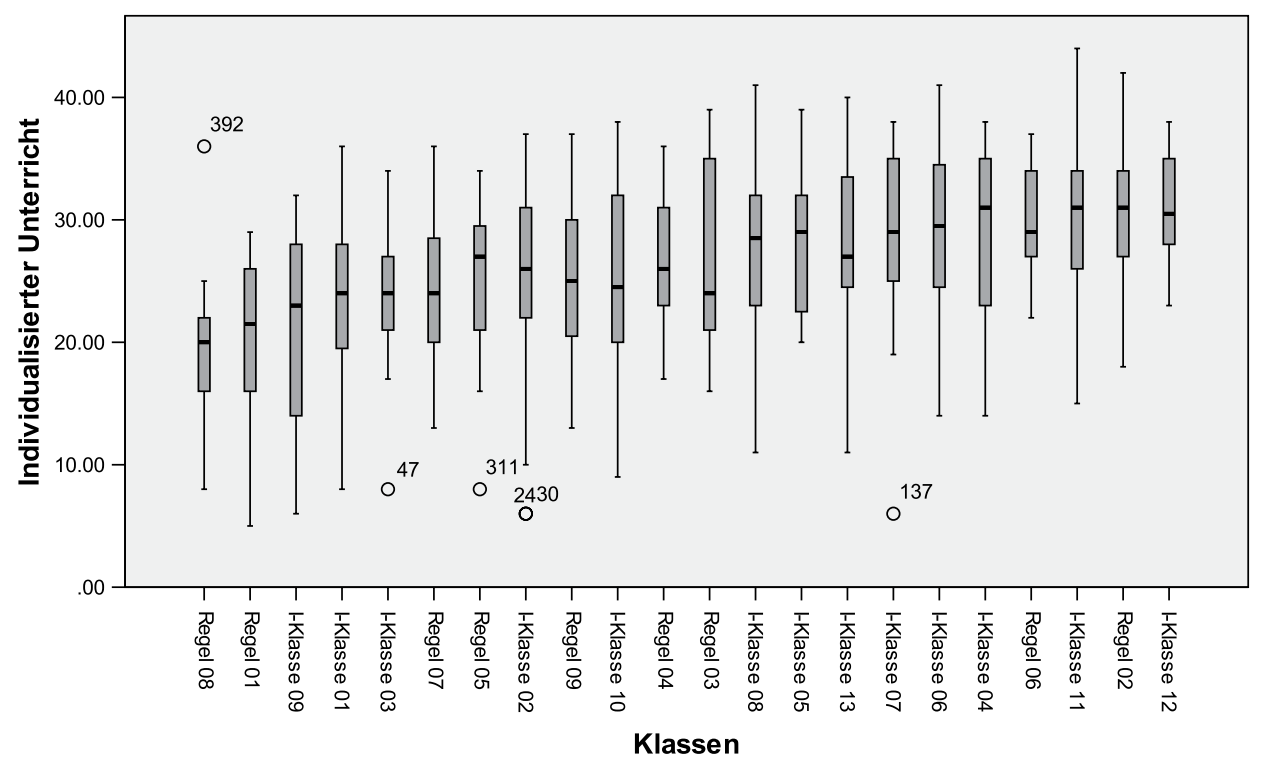
Abb. 1: Boxplots der einzelnen Klassen in der Skala „Individualisierter Unterricht“ geordnet nach den auf-
steigenden Rohwerten (Minimum=0; Maximum=44)

während die restlichen $84.11 \%$ durch die Personenunterschiede innerhalb der Klassen zu erklären sind. Verdeutlicht wird dies in der Abb. 1. Hier wurden die Klassen nach der Höhe ihrer Bewertung dargestellt. Es ist zu erkennen, dass sich nicht nur die einzelnen Schülerinnen und Schüler innerhalb einer Klasse (Streuung der einzelnen Boxplots), sondern auch die Schülerinnen und Schüler zwischen den Klassen (zwischen den Boxplots) unterscheiden. Zwar sind die Integrationsklassen vorwiegend im oberen Bereich der bewerteten Skala, jedoch gibt es auch Integrationsklassen im unteren Bereich der Skala.

\section{Diskussion}

Die vorliegende Studie analysierte die Wahrnehmung des Unterrichts der Schülerinnen und Schüler der Sekundarstufe I in Integrationsklassen und Parallelklassen. Ziel der Studie war es, die von Feyerer (1998) durchgeführte Studie zu replizieren und das Messinstrument erneut zu prüfen. Die ursprüngliche Skalenstruktur der Analyse von Feyerer (1998) konnte dabei nicht repliziert werden. Vielmehr zeigte sich in der vorliegenden Studie, dass zwar dieselbe Anzahl an Faktoren gefunden wurde, die Itemzuordnung zu den Faktoren war jedoch deutlich unterschiedlich zu jener von Feyerer ist. Die Faktoren „Unterschiedliche Aufgabenstellung“ und „Individuelles Eingehen auf unterschiedliche Schüler" entspricht weitgehend den von Feyerer (1998) gefundenen Inhalten. Der Faktor „Offene Lernformen“ konnte nicht repliziert werden, da drei Items aufgrund fehlender Reliabilität gekürzt werden mussten. Als Ursache für mangelnde Skalenreliabilität konnten jene Items identifiziert werden, welche eine negative Polung aufwiesen. Ein Grund hierfür war, dass die Polung dieser Items bei vielen Schülerinnen und Schülern zu Ver- 
ständnisproblemen geführt haben. Neben der negativen Polung könnte eine Ursache darin liegen, dass die Schülerinnen und Schüler die Methodik des offenen Unterrichts auf einer Likertskala von „stimmt nie“ bis „stimmt immer“ zu bewerten hatten. Vermutlich wäre ein Antwortformat mit zeitlichen Kategorien „einmal im Monat“ bis „täglich“ besser geeignet. Aus diesem Grund sollte die Subskala „Offene Lernformen“ in zukünftigen Erhebungen durch weitere positiv formulierte Items verlängert werden und das Antwortformat überdacht werden. Der hier gefundene dritte Faktor der Schülerorientierung sollte ebenfalls in zukünftigen Forschungen weiter analysiert werden, da dieser Faktor auch in der Literatur als Schlüssel für gelingenden inklusiven Unterricht gesehen wird (Dumke 1991; Heimlich 2007; Dyson 2010).

Aus den Items von Feyerer wurde zudem eine Kurzskala neu konstruiert. Diese wies eine ähnliche Reliabilität von 0,73 wie die Gesamtskala von Feyerer (1998) auf. Insofern konnte gezeigt werden, dass individueller Unterricht auch bei Kindern mit und ohne SPF ausreichend reliabel messbar ist. Da jedoch die vorliegende Studie die ursprüngliche Skalenstruktur von Feyerer nicht nachweisen konnte, erscheint es notwendig, in weiteren Studien die Skala konfirmatorisch zu bestätigen. Ein weiterer wichtiger Punkt wäre hier, die Schülerinnen- und Schülerbewertungen mit den Bewertungen der Lehrkräfte zu vergleichen. Hier würde sich eine verhaltensnahe Messung anbieten, bei der die Lehrerinnen und Lehrer nach den im Untersuchungsplan festgelegten Unterrichtsstunden angeben, mit welcher Methode die Unterrichtsstunde praktiziert wurde. Zudem wäre es für Schülerinnen und Schüler auch einfacher, Fragen anhand konkreter Beispiele zu beantworten. Dadurch wäre auch sichergestellt, dass alle Schülerinnen und Schüler dasselbe unter den Methoden verstehen (vgl. Mayr 2001).

Inhaltlich kann insgesamt festgestellt werden, dass die befragten Schülerinnen und Schüler angeben, dass individualisierter Unterricht eher häufig stattfindet. Der empirische Mittelwert lag bei den meisten Items über dem theoretischen Skalenmittelwert. Hinsichtlich der statistischen Vergleiche zwischen Integrations- und Regelschulklassen konnte auch für diese Untersuchung festgestellt werden, dass die Schülerinnen und Schüler in Integrationsklassen den eigenen Unterricht in einem höheren Ausmaß den Prinzipien der inklusiven Pädagogik entsprechend wahrnehmen, als Schülerinnen und Schüler in Regelschulklassen. Insofern konnten die Ergebnisse von Feyerer (1998) in dieser Stichprobe, welche auch Schülerinnen und Schüler mit SPF mit einbezieht, repliziert werden. Besonders deutliche Unterschiede waren bei den Items zur inneren Differenzierung im Unterricht zu finden, während die Items ,zum individuellen Eingehen der Lehrerinnen und Lehrer auf die Schülerinnen und Schüler" auch in den Regelschulklassen ähnlich positiv eingeschätzt wurden.

Bei der Auswertung auf Klassenebene wurde ersichtlich, dass sich auch die einzelnen Klassen voneinander unterscheiden. So gab es einzelne Integrations- und Regelklassen, welche die Schülerinnen und Schüler wahlweise als hoch oder niedrig bewerteten. Es findet somit auch nicht in allen Integrationsklassen ein ,optimaler', individualisierter Unterricht statt. Gerade die Integrationsklassen mit einem niedrigeren Wert in der Skala „Individualisierter Unterricht“" weisen einen Handlungsbedarf in der Aus- und Fortbildung von Lehrerinnen und Lehrer auf. Denn ein erfolgreicher gemeinsamer Unterricht von Kindern mit und ohne SPF entsteht nicht von selbst, sondern erfordert eine gelungene Kooperation von allen Lehrerinnen und Lehrern sowie eine gemeinsame Didaktik, wel- 
che auf die Bedürfnisse aller Kinder eingeht. Für die weitere Forschung wäre es wichtig, das Konzept eines inklusiven Unterrichts in landesweiten Studien (Large Scale Studien) mit zu erfassen. Dadurch können Unterschiede auf Ebene der Klasse sinnvoll interpretiert werden und weitere Aussagen zur Verbesserung des inklusiven Unterrichts getroffen werden. So sollte auch die Inklusion Teil des nationalen Bildungsmonitorings sein.

\section{Literatur}

Bühner, M. (2011). Einführung in die Test- und Fragebogenkonstruktion (3. Aufl.). München: Pearson Studium.

Bundesministeriums für Unterricht, Kunst und Kultur (BMUKK). (2008a). Richtlinien für die Umsetzung und das Monitoring von Qualitätsstandards im integrativen Unterricht von Schülerinnen und Schülern mit sonderpädagogischem Förderbedarf. http://www.bmukk.gv.at/ medienpool/17197/2008_18_beilage.pdf.

Bundesministeriums für Unterricht, Kunst und Kultur (BMUKK). (2008b). Richtlinien für Differenzierungs- und Steuerungsmaßnahmen im Zusammenhang mit der Feststellung des sonderpädagogischen Förderbedarfs. http://www.bmukk.gv.at/medienpool/17196/2008_19_beilage. pdf.

Bundesministeriums für Unterricht, Kunst und Kultur (BMUKK). (2009). Richtlinien für die Anwendung von Individuellen Förderplänen als Instrument der Unterrichtsplanung, Evaluierung und Qualitätssicherung im Unterricht von Schülerinnen und Schülern mit sonderpädagogischem Förderbedarf. http://www.bmukk.gv.at/medienpool/17699/2009_06_beilage.pdf.

Bundschuh, K., Heimlich, U., Klein, F., \& Krawitz, R. (Hrsg.). (2002a). Wörterbuch Heilpädagogik. Ein Nachschlagewerk für Studium und pädagogische Praxis (2. Aufl.). Bad Heilbrunn: Klinkhardt.

Dyson, A. (2010). Die Entwicklung inklusiver Schulen. Drei Perspektiven aus England. Die deutsche Schule, 102(2), 115-126.

Dumke, D. (1991). Soziale Kontakte behinderter Schüler in Integrationsklassen. Heilpädagogische Forschung, 1, 21-32.

Eder, F., \& Mayr, J. (1994). LFSK 4-8. Linzer Fragebogen zum Schul- und Klassenklima für die 4. bis 8. Klassenstufe. Göttingen: Hogrefe.

Erten, O., \& Savage, R. S. (2012). Moving forward in inclusive education research. International Journal of Inclusive Education, 16, 221-233.

Feyerer, E. (1998). Behindern Behinderte? Integrativer Unterricht auf der Sekundarstufe I. Innsbruck: Studien-Verlag.

Feyerer, E., \& Prammer, W. (2003). Gemeinsamer Unterricht in der Sekundarstufe I. Anregungen für eine integrative Praxis (Beltz Sonderpädagogik). Weinheim: Beltz.

Gasteiger Klicpera, B., \& Wohlhart, D. (2012). Arbeitspapier zur Umsetzung der UN-Behindertenrechtskonvention im Bildungssystem. Inklusive Regionen. Arbeitspapier für den 3. Runden Tisch zur Umsetzung der UN-Konvention über die Rechte von Menschen mit Behinderungen im schulischen Bereich (Bundesministeriums für Unterricht, Kunst und Kultur (BMUKK), Hrsg.). Wien.

Gebhardt, M., Gastager, A., Kernbichler, G., \& Gasteiger Klicpera, B. (2012a). Wirksamkeit von kooperativen Lernarrangements im Fach Deutsch der Schulstufen 5 und 6. Methodische Darstellung des Projekts KoopLea und erste Ergebnisse. http://www.oefeb2012.at.

Gebhardt, M., Schwab, S., Krammer, M., \& Gasteiger Klicpera, B. (2012b). Achievement and integration of students with and without Special Educational Needs (SEN) in the fifth grade. Journal of Special Education and Rehabilitation, 13(3-4), 7-19. 
Heimlich, U. (2007). Didaktik des gemeinsamen Unterrichts. In J. Walter \& F. B. Wember (Hrsg.), Handbuch Sonderpädagogik (Handbuch Sonderpädagogik, Bd. 2, S. 357-375). Göttingen: Hogrefe.

Heimlich, U., \& Jacobs, S. (2001). Integrative Schulentwicklung im Sekundarbereich. Das Beispiel der Integrativen Gesamtschule Halle/Saale. Bad Heilbrunn: Klinkhardt.

Helmke, A. (1988). Leistungssteigerung und Ausgleich von Leistungsunterschieden in Schulklassen. Unvereinbare Ziele? Zeitschrift für Entwicklungspsychologie und pädagogische Psychologie, 20, 45-76.

Hinz, A. (2002). Von der Integration zur Inklusion - terminologisches Spiel oder konzeptionelle Weiterentwicklung? Zeitschrift für Heilpädagogik, 53(9), 354-361.

Huber, C. (2006). Soziale Integration in der Schule?! Eine empirische Untersuchung zur sozialen Integration von Schülern mit sonderpädagogischem Förderbedarf im Gemeinsamen Unterricht. Marburg: Tectum-Verl.

Huber, C., \& Wilbert, J. (2012). Soziale Ausgrenzung von Schülern mit sonderpädagogischem Förderbedarf und niedrigen Schulleistungen im gemeinsamen Unterricht. Empirische Sonderpädagogik, 3(2), 147-165.

Klicpera, C., \& Gasteiger Klicpera, B. (2003). Soziale Erfahrungen von Grundschülern mit sonderpädagogischem Förderbedarf in Integrationsklassen. Zeitschrift für Heilpädagogik, 24, 61-71.

Klicpera, C., \& Gasteiger Klicpera, B. (2004). Prozess der Antragstellung auf einen sonderpädagogischen Förderbedarf. Ergebnisse einer Befragung der Bezirksschulinspektoren und der Leiter der sonderpädagogischen Zentren in 3 Bundesländern. Behindertenpädagogik, 43, 377-391.

Mayr. (2001). Innere Differenzierung auf der Sekundarstufe I: Eine Bestandsaufnahme. In F. Eder, G. Grogger, \& J. Mayr (Hrsg.), Sekundarstufe I. Probleme, Praxis, Perspektiven (S. 218-237). Innsbruck: Studienverlag.

Preuss-Lausitz, U. (1997). Wohnortnahe Integration. Gemeinsame Erziehung behinderter und nichtbehinderter Kinder in der Uckermark- Grundschule in Berlin. In P. Heyer, U. Preuss-Lausitz, \& J. Schöler (Hrsg.), ,, Behinderte sind doch Kinder wie wir! “. Gemeinsame Erziehung in einem neuen Bundesland (1. Aufl.). Berlin: Wiss.-und-Technik-Verl.

Rosenberger, M. (1998). Schule ohne Aussonderung. Ideen, Konzepte, Zukunftschancen. Neuwied: Leuchterhand.

Rutte, V., \& Schönwiese, V. (2000). Zur Geschichte und Situation der Integration in Österreich. In A. Ginnold \& M. Hans (Hrsg.), Integration von Menschen mit Behinderung. Entwicklungen in Europa (1. Aufl., S. 205-221). Neuwied: Luchterhand.

Sander, A. (2005). Liegt Inklusion im Trend? Vierteljahresschrift für Heilpädagogik und ihre Nachbargebiete (VHN), 75, 51-53.

Schulze, M. (2013). Inklusive Bildung - Menschenrechtliche Grundlagen. In: S. Schwab, M. Gebhardt, E. M. Ederer-Fick, \& B. Gasteiger-Klicpera (Hrsg.), Theorien, Konzepte und Anwendungsfelder der inklusiven Pädagogik (S. 11-23). Wien: Facultas.

Schwab, S., Gebhardt M., \& Gasteiger-Klicpera, B. (2013). Predictors of social inclusion of students with and without SEN in integrated settings. Croatian Review of Rehabilitation Research, 1, 106-114.

Seitz, S., \& Scheidt, K. (2012). Vom Reichtum inklusiven Unterrichts - Sechs Ressourcen zur Weiterentwicklung. Zeitschrift für Inklusion. 1-2. http://www.inklusion-online.net/index.php/ inklusion/article/view/148/140.

Snijders, T. A. B., \& Bosker, R. (2011). Multilevel analysis. An introduction to basic and advanced multilevel modeling. [S.1.]. Sage Publications.

Specht, W. (1991). Perspektiven wissenschaftlicher Begleitforschung im Bereich der Schulversuche zur Integration behinderter Kinder. Graz: Zentrum für Schulversuche und Schulentwicklung.

Statistik Austria. (2013). Bildung in Zahlen. Tabellenband. Wien: Verlag Österreich.

United Nations. (2006). Convention of the Rights of Persons with Disabilities (CRPD). New York. 